The final publication is available at Elsevier via http://dx.doi.org/10.1016/j.clae.2014.09.007 ( 2014. This manuscript version is made available under the CC-BY-NC-ND 4.0 license http://creativecommons.org/licenses/by-nc-nd/4.0/

\title{
1 Application of a new grading scale for tear ferning in non-dry eye and dry eye 2 subjects
}

$4 \quad$ Ali Masmali ${ }^{\mathrm{a}, *}$, Sultan AL-Qhtani ${ }^{\mathrm{a}}$, Talha M. Al-Gasham ${ }^{\mathrm{a}}$, Gamal A. El-Hiti ${ }^{\mathrm{a}}$, Christine

5 Purslow ${ }^{\mathrm{b}}$, Paul J. Murphyc

${ }^{a}$ Cornea Research Chair (CRC), Department of Optometry, College of Applied Medical

8 Sciences, King Saud University, P.O. Box 10219, Riyadh 11433, Saudi Arabia

$9{ }^{b}$ School of Optometry and Vision Sciences, Cardiff University, Cardiff, UK

c School of Optometry and Vision Science, University of Waterloo, Waterloo, Canada

12 The authors have no conflicts of interest to disclose.

* Corresponding author at: Cornea Research Chair (CRC), Department of Optometry, College of 15 Applied Medical Sciences, King Saud University, P.O. Box 10219, Riyadh 11433, Saudi Arabia. 16 Tel.: +966 11 4693547; fax: +966 114693536

17 E-mail address: amasmali@ksu.edu.sa (A. Masmali).

19 Number of Tables: 6

21 Number of Figures: 5

23 Fund has been received from the College of Applied Medical Sciences Research Canter and the 24 Deanship of Scientific Research at King Saud University. 
27 Purpose: To apply the Masmali tear ferning (TF) grading scale on non-dry eye (NDE) and dry 28 eye (DE) subjects to test the validity of the grading scale in practice, and to describe the grading 29 scale range for NDE and DE.

30

31

32

33

34

35

36

37 6 7

Method: Forty NDE subjects (20 males, 20 females) and 40 DE subjects (23 males, 17 females) ranging in age from 19 to 53 years (mean \pm SD: $25.3 \pm 5.5$ ) with no other ocular disease, no contact lens wear, and not pregnant or breastfeeding were recruited. McMonnies scores were used for subject grouping. Phenol red thread (PRT) and slit-lamp test were used. A tear sample was collected from right eye, which was then dried to produce a ferning pattern, that was observed using a digital microscope, and graded.

Results: Mean McMonnies, PRT and TF grade in NDE subjects were 7.1 $\pm 3.8,27.4 \pm 4.3 \mathrm{~mm}$ and 0.78 \pm 0.40 , respectively. Median McMonnies, PRT and TF grade in DE subjects were $16.5 \pm 3.0$, $9.0 \pm 2.0 \mathrm{~mm}$ and $2.3 \pm 1.48$, respectively. In NDE subjects, grades 0.0 to 1.8 were observed $(82.5 \%$ Grade 0.0 to 1.00 ). Grades 2.0 to 4.0 were observed in DE subjects (72.5\% Grades 2.0 to 3.0 ). For all subjects, there were large correlations between TF grade and PRT $(r=-0.79)$, PRT and McMonnies ( $r=-0.60)$, and TF and McMonnies $(r=0.73)$.

Conclusions: The Masmali TF grading scale showed good validity in describing the TF patterns. Grades $\geq 2$ can be classified as abnormal patterns. The TF test has the potential to be used in the clinic.

Keywords: Tear ferning; dry eye disease; Masmali grading scale; phenol red thread test 


\section{Introduction}

The tear film has three main functions: maintenance of ocular surface health, creation of

51 a smooth optical surface for clear vision, and protection of the eye from foreign bodies and

52 infection. It consists of three main layers [1-4]. The outermost layer of the tear film is the lipid

53 layer. This layer acts to limit evaporation of the aqueous component, as well as providing

54 lubrication for eyelid movement and a smooth optical surface [5]. The middle layer consists

55 principally of water, which contains ions and nutrients to supply the corneal and conjunctival

56 epithlelia, as well as immunological proteins for ocular defence against infections [6]. The

57 innermost layer consists of mucous secreted from conjunctival goblet cells and from the corneal

58 epithelia, which provides a foundational glycocalyx over the ocular surface to which the tear film

59 can adhere [2].

Dry eye (DE) is a multifactorial disease of the ocular surface and the tears that interferes

61 with the normal production and function of the tear film, and which leads to decreases in tear

62 stability and tear volume, and increases in evaporation and tear osmolarity [7,8]. It can also lead

63 to damage to the ocular surface, visual disturbance, and discomfort [8,9]. It has been reported

64 that DE can affect quality of life in different ways. Symptoms of irritation, effects on visual

65 function or performance are all signs and symptoms that can affect quality of life of DE patients

66 [8]. Blurry vision, hyperaemia, mucoid discharge, ocular irritation and dryness are the most

67 common complaints associated with $\mathrm{DE}$ [8]. The diagnosis of $\mathrm{DE}$ is complicated due to the

68 multifactorial etiology. In particular, the small tear film volume limits potential analysis of the

69 tear film composition, which may contain immunological markers for DE [8]. Tear film

70 composition analysis is also very challenging for clinicians and researchers because of the

71 dynamic and transparent nature of tears [10-12]. Various methods have been developed to test 
72 some aspect of the tear film, such as Schirmer's test [13-15], phenol red thread (PRT) test

73 [16-19], tear osmolarity [20-23], tear meniscus height [24,25] and tear break-up time (TBUT)

74 [26]. Each test assesses some aspect of the tear film, but no single test is able to definitively

75 diagnose dry eye. Instead a combination of tests is employed to provide a final diagnosis [8].

76 In defining dry eye, the Dry Eye Workshop reports $(1999,2007)$ emphasised the role of

77 tear osmolarity within a model of the disease process cycle. Due to either an under-production of

78 tears or to increased tear evaporation, the salt concentration of the tear film increases, creating an

79 osmotic stress for the ocular surface epithelia. This leads to an inflammatory response for the

80 ocular surface, which further alters the tear film, leading to a reinforcing cycle of increasing

81 osmolarity and inflammation [8]. Analysis of the tear osmolarity is therefore a priority for dry

82 eye disease management, and the TearLab Osmolarity System (TearLab Corporation, San Diego,

83 USA) uses 'lab on a chip' technology to provide an in-clinic assessment [27].

A potential alternative to instrument-based osmolarity testing is to use the phenomenon

85 of tear ferning. Tear ferning involves the drying of a tear sample on a glass slide at normal room

86 temperature and humidity conditions to produce a fern, a crystallization pattern that can be

87 inspected by the use of a light microscope [28-30]. The test can be performed within the clinic

88 quickly and cheaply with the advantage that the tear film chemical properties, especially

89 electrolytes and large molecules, like proteins, can be so investigated indirectly [31]. Since the

90 tear film is a complex solution and has different organic and inorganic components, variation in

91 its composition or concentration will produce changes to the tear fern pattern [30,32].

The pattern variation has been suggested as a simple test for the quality of the tear film,

93 with the potential of being used in the optometrist's clinic [28,33] and has shown good

94 sensitivity and specificity [33-35], and repeatability [31]. To assist in assessing the ferning 
95 patterns, Rolando developed a tear ferning pattern grading scale [29]. The Rolando scale 96 categorises the observed tear ferning pattern into four grades, known as Type I, II, III and IV

97 [29]. It was found that Types I and II were common within healthy eyes and Types III and IV 98 were common within kerato-conjunctivitis sicca eyes [29]. Very recently, a new grading scale

99 for the tear ferning pattern has been developed to overcome some of the limitations associated 100 with Rolando scale, such as the overlap between grades [31, 36]. The new 5-point Masmali 101 grading scale can be used by both researcher and clinician when using the tear ferning test to 102 investigate dryness of the eye.

103 Grade 0 has the full phenomenon of ferning pattern, with no spaces or gaps between the 104 ferns and branches; the density of ferns and branches is decreased in Grade 1 with the 105 appearance of small spaces and gaps between the ferns and branches. These ferns and branches 106 are decreased to become thick and large with the presence of clear spaces and gaps in Grade 2. 107 The spaces and gaps are increased in Grade 3 with no ferns, but with the presence of large 108 crystals. The phenomenon of ferning pattern is totally absent in Grade 4. A visual presentation of 109 the 5 grades has been published in Masmali et al [31].

110 The aim of this research was to apply the new Masmali TF grading scale on tear samples 111 from subjects with NDE and DE to test the validity of the grading scale in practice, and to 112 describe the grading scale range for NDE and DE for the first time using this scale.

\section{2. Methods}

115 Eighty subjects (40 non-dry eyes (NDE): 20 males and 20 females; 40 dry eyes (DE): 23 116 males and 17 females), who ranged in age from 19 to 53 years (mean \pm SD: $25.3 \pm 5.5$ years) with 117 no symptoms of any other ocular disease, who did not wear contact lenses, and were not 
118

119

120

121

122

123

124

125

126

127

128

129

130

131

132

133

134

135

136

137

138

139

140

pregnant or breastfeeding, were enrolled in the study. All subjects completed a McMonnies dry eye symptoms questionnaire and were grouped into a healthy or dry eye group according to their response to the McMonnies questionnaire. Dry eye was diagnosed for a score $>14.5$ [37-38].

Slit lamp assessment of the ocular surface and adnexa was performed first, followed by measurement of tear volume with the phenol red thread (PRT) test were further used to describe the two subject groups. The slit-lamp examination was performed to check the external and anterior part of the eye for the absence of any ocular disease.

PRT strips were purchased from ZONE-QUICK (Showa Yakuhin Kako Co, Ltd). A 3mm length of the thread was folded and inserted $1 / 3$ of the distance from the temporal canthus of the lower eyelid, with the eye in the primary position. The thread was gently removed after 15 seconds and the length of the discoloured portion was determined (mm). The test was applied to the both eyes, but the reading for the right eye only was used in analysis.

Tear samples $(1 \mu \mathrm{l})$ were collected from the lower meniscus of the right eye using glass

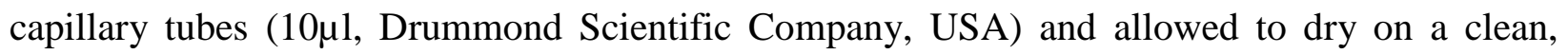
unused glass slide for 10 minutes under normal room temperature $\left(23^{\circ} \mathrm{C}\right)$ and humidity $(40 \%)$. Samples were immediately observed under digital microscope (Olympus DP72) with 10X magnification. Each ferning pattern observed was graded using the 5-point grading scale [31] in 0.1 increments to improve grade refinement [39].

The study design was masked to avoid any bias. The McMonnies questionnaire, slit-lamp examinations, PRT and tear collections were completed by one investigator, and the imaging of the tear ferning patterns slides and the grading of the ferning patterns was completed by another investigator who was blind to the subject dry eye classification and other test results. All subjects were examined at a morning visit between 8:30 to 11:30 am. 
142 Centre, King Saud University. The research followed the tenets of the Declaration of Helsinki, in

143 which informed consent was obtained from the subjects after explanation of the nature and 144 possible consequences of the study.

\section{$146 \quad 2.1 \quad$ Statistical analyses}

147 Data were collated using Excel (Microsoft Office 2010) and analysed using the SPSS 148 software (IBM Software, version 20). Data of tear ferning, PRT and McMonnies were examined 149 for normality using Kolmogorov-Smirnov tests and all 3 data sets were found to be normally 150 distributed (Kolmogorov-Smirnov, p >0.05) for NDE subjects and not normally distributed 151 (Kolmogorov-Smirnov, $\mathrm{p}<0.05)$ for DE subjects. The mean $\pm \mathrm{SD}$ was used to describe the 152 results from NDE subjects, while the median \pm IQR was used to describe the results for DE 153 subjects.

Spearman's correlation was used to investigate the relationship between all data obtained 155 from the three tests (PRT, McMonnies and TF). Pearson's correlation was used to study the 156 relationship between the tests in NDE subjects, while Spearman's correlation was used in DE 157 subjects. Correlation coefficients were graded as: small (0.10 to 0.29$)$, medium (0.30 to 0.49 ), 158 and large (0.50 to 1.00$)$ [40]. TF, PRT and McMonnies based on the TF scores. 


\section{Results}

The mean and median values for McMonnies questionnaire, phenol red thread test, and tear ferning grading scale are shown in Table 1.

Table 1. The mean \pm SD (NDE) and median \& IQR (DE) for McMonnies questionnaire scores, PRT test and TF grading scale

\begin{tabular}{lll}
\hline \multirow{2}{*}{ Test } & Mean \pm SD & Median (IQR) \\
\cline { 2 - 3 } & NDE & DE \\
\hline McMonnies questionnaire & $7.1 \pm 3.8$ & $16.5(15.0-18.0)$ \\
PRT (OD) & $27.4 \pm 4.3$ & $9.0(8.0-9.0)$ \\
TF grading scale (OD) & $0.8 \pm 0.4$ & $2.3(2.10-3.60)$ \\
\hline
\end{tabular}

171 samples within the range of 0.0 to 1.0. For the DE subjects, grades from 2.0 to 3.0 were observed in the majority (72.5\%) of samples, with the remaining $27.5 \%$ of subjects having TF grading scale range of 3.1 to 4.0. The frequency of grading for NDE and DE subjects is recorded in Tables 2 and 3, respectively. Samples of NDE and DE tear ferning images are shown in Figures

1771 and 2, respectively.

Table 2. The frequency of grading for NDE subjects on the TF grading scale

\begin{tabular}{lll}
\hline TF grading scale & Frequency & Percentage (\%) \\
\hline $0.0-0.5$ & 11 & 27.5 \\
$0.6-1.0$ & 22 & 55 \\
$1.1-1.5$ & 4 & 10 \\
$1.6-1.8$ & 3 & 7.5 \\
\hline
\end{tabular}




\begin{tabular}{lll}
\hline TF grading scale & Frequency & Percentage (\%) \\
\hline $2.0-2.5$ & 23 & 57.5 \\
$2.6-3.0$ & 6 & 15 \\
$3.1-3.5$ & 1 & 2.5 \\
$3.6-4.0$ & 10 & 25 \\
\hline
\end{tabular}

188

189

190

191

192

193

\section{Figures 1-2 here} and PRT test, and between PRT test and McMonnies score. There was negligible correlation between the TF and McMonnies scores.

In DE subjects (Table 5), there was a small negative correlation between TF grade and PRT test; and a medium negative correlation between TF grade and McMonnies score. There was also a negligible correlation between PRT test and McMonnies score.

When all subject data (Table 6) was grouped together, there was a large negative correlation between PRT and McMonnies score (Figure 3), and between PRT and TF grade (Figure 4). There was also a large positive correlation between TF grade and McMonnies score (Figure 5).

Table 4. Correlations between TF grade, PRT and McMonnies score in NDE subjects

\begin{tabular}{lllll}
\hline & & TF & PRT & McMonnies \\
\hline \multirow{3}{*}{ TF } & Pearson Correlation & 1.00 & -0.30 & 0.03 \\
& Sig. (2-tailed) & & 0.07 & 0.84 \\
& $\mathrm{~N}$ & 40 & 40 & 40 \\
\hline \multirow{3}{*}{ PRT } & Pearson Correlation & -0.30 & 1.00 & -0.32 \\
& Sig. (2-tailed) & 0.07 & & 0.04 \\
& $\mathrm{~N}$ & 40 & 40 & 40 \\
\multirow{3}{*}{ McMonnies } & Pearson Correlation & 0.03 & -0.32 & 1.00 \\
& Sig. (2-tailed) & 0.84 & 0.04 & \\
& $\mathrm{~N}$ & 40 & 40 & 40 \\
\hline
\end{tabular}



208

Table 5. Correlations between TF grade, PRT and McMonnies score in DE subjects

\begin{tabular}{lllll}
\hline & & TF & PRT & McMonnies \\
\hline \multirow{3}{*}{ TF } & Spearman's Correlation & 1.00 & -0.20 & -0.30 \\
& Sig. (2-tailed) & & 0.22 & 0.06 \\
& $\mathrm{~N}$ & 40 & 40 & 40 \\
\hline \multirow{3}{*}{ PRT } & Spearman's Correlation & -0.20 & 1.00 & 0.12 \\
& Sig. (2-tailed) & 0.22 & & 0.47 \\
& $\mathrm{~N}$ & 40 & 40 & 40 \\
\multirow{3}{*}{ McMonnies } & Spearman's Correlation & -0.30 & 0.12 & 1.00 \\
& Sig. (2-tailed) & 0.06 & 0.47 & 40 \\
\hline
\end{tabular}

Table 6. Correlation between TF grade, PRT and McMonnies score in all subjects’ data

\begin{tabular}{lllll}
\hline & & TF & PRT & McMonnies \\
\hline \multirow{3}{*}{ TF } & Spearman's Correlation & 1.00 & -0.79 & 0.73 \\
& Sig. (2-tailed) & & 0.00 & 0.00 \\
& N & 80 & 80 & 80 \\
\hline \multirow{3}{*}{ PRT } & Spearman's Correlation & -0.79 & 1.00 & -0.60 \\
& Sig. (2-tailed) & 0.00 & & 0.00 \\
& N & 80 & 80 & 80 \\
\multirow{3}{*}{ McMonnies } & Spearman's Correlation & 0.73 & -0.60 & 1.00 \\
& Sig. (2-tailed) & 0.00 & 0.00 & \\
& $\mathrm{~N}$ & 80 & 80 & 80 \\
\hline
\end{tabular}

Figures 3-5 here

For further analysis, all of the subjects were first of all divided into four groups based on the TF scores (Group I: 0-1; Group II: 1.1-2; Group III: 2.1-3; Group IV: 3.1-4) and secondly into two groups based on TF scores (NDE subjects: 0-1.8; DE subjects: 2-4). This second classification was based on a TF grade of 1.8, which was found to be the maximum TF grade in NDE subjects, while a grade of 2 was the minimum for DE subjects. The data for all subjects was not normally distributed and non-parametric tests were used.

There was a significant difference within the four groups (Kruskal-Wallis; p <0.01) and within the two groups (Kruskal-Wallis; p <0.01) for TF, PRT and McMonnies. A Mann-Whitney 
219 test found a significant difference between the NDE and DE groups in TF grade $(\mathrm{z}=-7.71 ; \mathrm{p}$ $220<0.01)$, PRT $(\mathrm{z}=-6.39 ; \mathrm{p}<0.01)$ and McMonnies score $(\mathrm{z}=-7.70 ; \mathrm{p}<0.01)$, based on the TF 221 grade.

222

\section{$223 \quad 4 . \quad$ Discussion}

The 5-point Masmali grading scale [31] was introduced to provide a reliable method of

225 tear fern grading that would overcome the limitations within Rolando’s grading scale, especially 226 the lack of sensitivity in categorisation of ferning patterns due to the overlap across Type I \& II 227 grades. In a previous published study, the 5-point scale was found to be discriminating, linear 228 and reliable [31].

This study has applied the 5-point grading scale on tear ferning patterns obtained from

230 NDE and DE subjects, using 0.1 increments, to examine the validity of the scale to differentiate

231 between these subject types. The use of 0.1 increments has been suggested to increase test 232 sensitivity [39]. In NDE subjects, 82.5\% were found to have a grade between 0.0 and 1.0, with 233 the remaining $17.5 \%$ to have grades from 1.1 to 1.8 . In sharp contrast, $72.5 \%$ of DE ferning 234 patterns were graded between 2.1 to 3.0 , and $27.5 \%$ graded from 3.1 to 4.0 . There was no 235 overlap in TF grading for this group of subjects, as classified using the McMonnies 236 questionnaire.

Statistical analysis of the results found a significant difference within the TF grades between NDE and DE subjects. When the subjects were classified into either two or four groups,

239 based on the tear ferning scores, there was always a significant difference in the PRT and 240 McMonnies score between the groups. This reverse analysis of the data indicates that the tear 
241 ferning test, based on the new grading scale, has the ability and sensitivity to differentiate 242 between NDE and DE subjects.

Taking the full data set for all subjects, the tear ferning grading scores had a large

244 correlation with both the PRT and McMonnies questionnaire results. A medium correlation was

245 found between TF grade and PRT in NDE subjects and between TF grade and McMonnies

246 scores in DE subjects. The moderate strength of these correlations may help in explaining the

247 poor relationship noted between ocular signs and symptoms in dry eye [41]. If dry eye is often,

248 but not always, associated with a deficient aqueous component volume [2], then changes in the 249 content of the aqueous component may be detectable with tear ferning. This hypothesis is 250 supported by the Chi-square test, which showed very strong evidence for a relationship between 251 tear ferning, PRT and McMonnies. While a previous study [42] reported little relationship 252 between TF score and other tear film tests, this may be due to limitations in the grading scale 253 used. In contrast, the use of the new tear ferning grading scale in this study has allowed a 254 stronger correlation with some tests to be identified. All of these results support the role of tear 255 ferning as a useful diagnostic test, with the potential to work well with other diagnostic tests.

256 Based on the statistical analysis, we propose that a tear ferning pattern Grade $\geq 2$ can be 257 taken as a cut-off grade between non-dry eye and abnormal, with any ferning pattern less than 258 Grade 2 considered as representing a non-dry eye tear film. This classification will help support 259 practitioners to grade and evaluate the ocular tear ferning patterns.

Tear ferning test is a simple and inexpensive test, which has features that make it suitable 261 for application in the eye clinic when evaluating the tear film [31], and the new 5-point tear fern 262 grading scale has the ability to distinguish between non-dry eye and dry eye subjects. 
264 support the role of this test in the clinic and to help in the diagnosis and management of dry eye

265 disease. The next useful step is to compare the chemical analysis of the collected tear sample 266 with its tear ferning pattern, matching it with the grading scale.

267

$268 \quad$ 5. Conclusions

269 A tear ferning Grade $\geq 2$ can be classified as representing an abnormal pattern. The 270 Masmali 5-point TF grading scale has shown good validity. The tear ferning test can be used as a 271 clinical and research method to detect the dryness of the eye and investigate the tear film along 272 with other additional tests.

273

274 Disclosure

275 None of the authors has any proprietary interest in this manuscript.

276

277 Acknowledgements

The authors extend their appreciation to the College of Applied Medical Sciences 279 Research Centre and the Deanship of Scientific Research at King Saud University for its funding 280 of this research.

281 


\section{References}

287 1. McGinnigle S, Naroo SA, Eperjesi F. Evaluation of dry eye. Surv Ophthalmol $288 \quad$ 2012;57:293-316.

289 2. Johnson ME, Murphy PJ. Changes in the tear film and ocular surface from dry eye 290 syndrome. Prog Retin Eye Res 2004;23:449-474.

291 3. Holly FJ, Lemp MA. Tear physiology and dry eyes. Surv Ophthalmol 1977;22:69-87.

292 4. Wolff E. Mucocutaneous junction of lid margin and distribution of tear fluid. Trans 293 Ophthal Soc UK 1946;66:291-308.

294 5. Craig JP, Tomlinson A. Importance of the lipid layer in human tear film stability and 295 evaporation. Optom Vis Sci 1997;74:8-13.

296 6. Bachman WG, Wilson G. Essential ions for maintenance of the corneal epithelial surface. 297 Invest Ophthalmol Vis Sci 1985;6:1484-1488.

298 7. Murube J. Tear osmolarity. Ocul Surf 2006;1:62-73.

299 8. The definition and classification of dry eye disease: report of the Definition and 300 Classification. Subcommittee of the International Dry Eye Workshop. Ocul Surf $301 \quad 2007 ; 5: 75-92$.

302 9. Colligris B, Alkozi HA, Printor J. Recent developments on dry eye disease treatment 303 compounds. Saudi J Ophthalmol 2014;28:19-30.

304 10. Pearce EI, Tomlinson A. Spatial location studies on the chemical composition of human 305 tear ferns. Ophthal Physl Opt 2000;20:306-313.

306 11. Li M, Du C, Zhu D, Shen M, Cui L, Wang J. Daytime variations of tear osmolarity and 307 tear meniscus volume. Eye Contact Lens. 2012;38:282-287. 
308 12. Versura P, Profazio V, Campos EC. Performance of tear osmolarity compared to previous diagnostic tests for dry eye diseases. Curr Eye Res 2010;35:553-564.

310 13. Saleh TA, McDermott B, Bates Ak, Ewings P. Phenol red thread test vs Schirmer's test: a comparative study. Eye 2006:913-915.

312 14. Bawazeer AM, Hodge WG. One-minute Schirmer test with anesthesia. Cornea $313 \quad 2003 ; 22: 285-287$.

314 15. Vashisht S, Singh S. Evaluation of phenol red thread test versus Schirmer test in dry 315 eyes: a comparative study. Int J Appl Basic Med Res 2011;1:40-42.

316 16. Wee SW, Chun YS, Moon NJ, Kim JC. Clinical usefulness of the phenol red thread test as diagnostic tool in dry eye patient. J Korean Ophthalmol Soc 2012;53:193-199.

318 17. Miller WL, Doughty MJ, Narayanan S, Leach NE, Tran A, Gaume AL, Bergmanson JP. 319

322 18. Tomlinson A, Blades KJ, Pearce EI. What does the phenol red thread test actually 323 measure? Optom Vis Sci 2001;78:142-146.

324 19. Masmali, A, Alqahtani TA, Alharbi A, El-Hiti GA. Comparative study of repeatability of 325 phenol red thread test versus Schirmer's test in normal adults in Saudi Arabia. Eye 326 Contact Lens 2014;40:127-131.

327 20. Tomlinson A, Khanal S, Ramaesh K, Diaper C, McFadyen A. Tear film osmolarity: 328 determination of a referent for dry eye diagnosis. Invest Ophthalmol Vis Sci 329 2006;47:4309-4315. 
330 21. Szalai E, Berta A, Szekanecz Z, Szûcs G, Módis L. Evaluation of tear osmolarity in nonSjögren and Sjögren syndrome dry eye patients with the Tear Lab system. Cornea 2012;31:867-871.

333 22. Lemp MA, Bron, AJ, Baudouin C, Benítez Del Castilo JM, Geffen D, Tauber J, Foulks GN, Pepose JS, Sullivan D. Tear osmolarity in the diagnosis and management of dry eye 335 disease. Am J Ophthalmol 2011;151:792-798.

336 23. Masmali A, Alrabiah S, Alharbi A, El-Hiti GA, Almubrad T. Investigation of tear 337 osmolarity using the TearLab ${ }^{\mathrm{TM}}$ osmolarity system in normal adults in Saudi Arabia. Eye Contact Lens 2014;40:74-78.

339 24. García-Resúa C, Santodomingo-Rubido J, Lira M, Giraldez MJ, Vilar EY. Clinical 340 assessment of the lower tear meniscus height. Ophthalmol Physiol Opt 2009;29:487-496.

341 25. Ibrahim OM, Dogru M, Takano Y, Satake Y, Wakamatsu TH, Fukagawa K, Tsubota K, 342 Fujishima H. Application of Visante optical coherence tomography tear meniscus height 343 measurement in the diagnosis of dry eye disease. Ophthalmology 2010;117:1923-1929.

344 26. Cho P, Ho K-Y, Huang Y-C, Chui H-Y, Kwan M-C. Comparison of non-invasive tear 345 break-up time measurements from black and white background instruments. Optom 346 Vision Sci 2004;81:436-441.

347 27. Benelli U, Nardi M, Posarelli C, Albert T. Tear osmolarity measurement using the 348 349 Tearlab $^{\mathrm{TM}}$ Osmolarity System in the assessment of dry eye treatment effectiveness. Cont Lens Anterior Eye 2010;33:61-67.

350 28. Tabbara KF, Okumoto M. Ocular ferning test. A qualitative test for mucus deficiency. 351 Ophthalmology 1982; 89:712-714. 
352 29. Rolando M. Tear mucus ferning test in normal and keratoconjunctivitis sicca eyes. $353 \quad$ Chibret Int J Ophthalmol 1984;2:32-41.

354 30. Golding TR, Brennan NA. The basis of tear ferning. Clin Exp Optom 1989;72:102-112.

355 31. Masmali AM, Murphy PJ, Purslow C. Development of a new grading scale for tear 356 ferning. Cont Lens Anterior Eye 2014;37:178-184.

357 32. Kogbe O, Liotet S, Tiffany JM. Factors responsible for tear ferning. Cornea $358 \quad 1991 ; 10: 433-444$.

359 33. Vaikoussis E, Georgiou P, Nomicarios D. Tear mucus ferning in patients with Sjogren's 360 syndrome. Doc Ophthalmol 1994;87:145-151.

361 34. Ravazzoni L, Ghini C, Macri A, Rolando M. Forecasting of hydrophilic contact lens 362 tolerance by means of tear ferning test. Graefes Arch Clin Exp Ophthalmol $363 \quad 1998 ; 236: 354-358$.

364 35. Norn M. Quantitative tear ferning. Clinical investigations. Acta Ophthalmol $365 \quad$ 1994;72:369-372.

366 36. Masmali AM. Development of a tear ferning test protocol and a new grading scale. PhD 367 thesis, Cardiff University, 2010.

368 37. McMonnies CW, Ho A. Responses to a dry eye questionnaire from a normal population. $369 \quad$ J Am Optom Assoc 1987;58:588-591.

$37038 . \quad$ Nichols KK, Nichols JJ, Mitchell G, Lynn M. The reliability and validity of McMonnies 371 dry eye index. Cornea 2004;23:365-371.

372 39. Bailey IL, Bullimore MA, Raasch TW, Taylor HR. Clinical grading and the effects of 373 scaling. Invest Ophthalmol Vis Sci 1991;32:422-432. 
374 40. Cohen JW. Statistical power analysis for the behavioral sciences. Hillsdale NJ, Lawrence $375 \quad$ Erlbaum Associates, 1988.

376 41. Nichols KK, Nichols JJ, Mitchell GL. The lack of association between signs and symptoms in patients with dry eye disease. Cornea 2004;23:762-770.

378

379 42. Evans KS, North RV, Purslow C. Tear ferning in contact lens wearers. Ophthal Physiol 380 Opt 2009;29:199-204.

381 


\section{Figures Captions}

383 Figure 1. Sample of tear ferning pattern obtained from a NDE subject (equivalent to Grade 0).

384 Figure 2. Sample of tear ferning pattern obtained from a DE subject (equivalent to Grade 3).

385 Figure 3. Correlation between PRT test and McMonnies score for all subjects.

$386 \quad$ Figure 4. Correlation between tear ferning grade and PRT test for all subjects.

387 Figure 5. Correlation between tear ferning grade and McMonnies score for all subjects. 


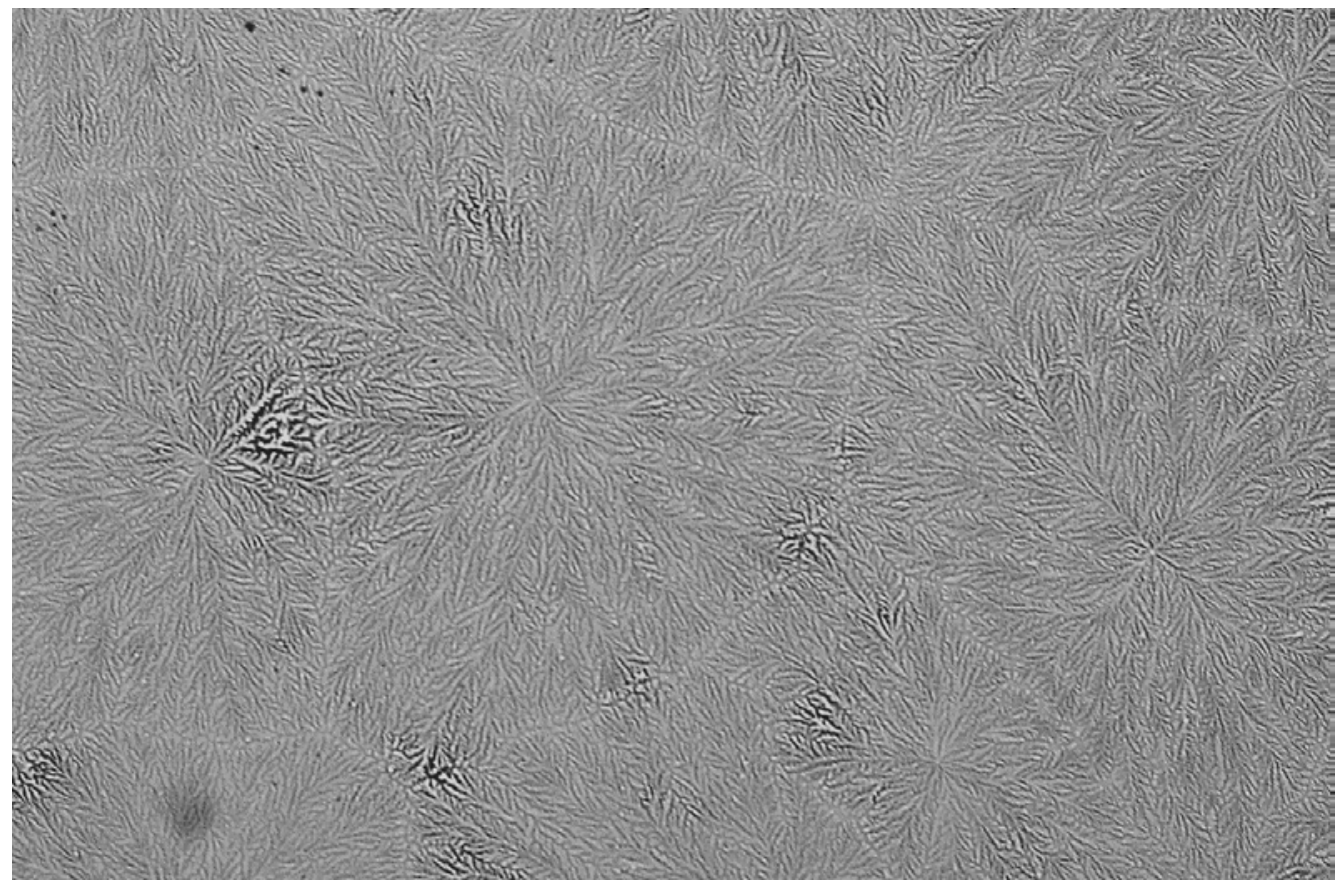

392 Figure 1. Sample of tear ferning pattern obtained from a healthy eye subject (equivalent to 393 Grade 0).

394

395 


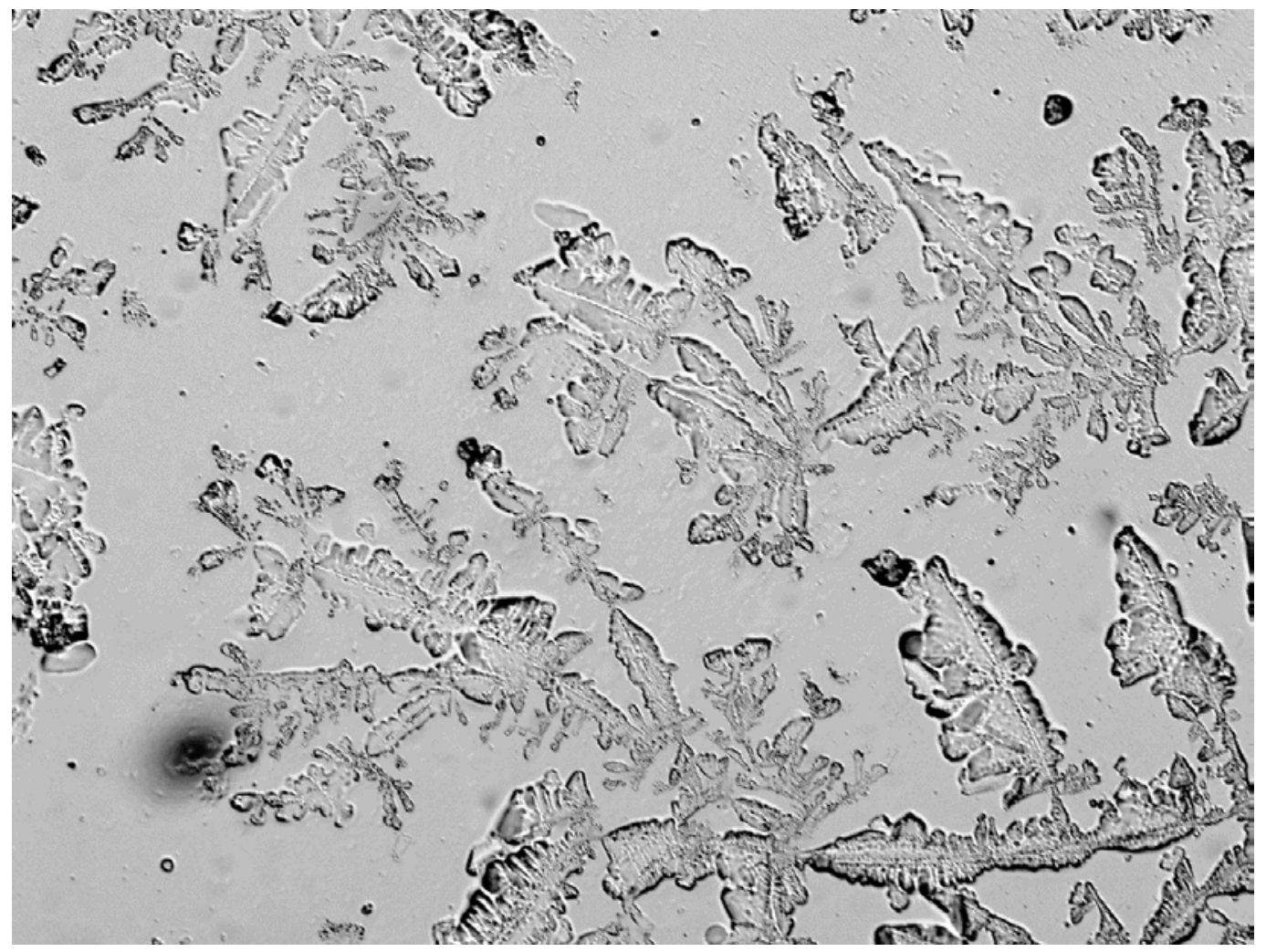

Figure 2. Sample of tear ferning pattern obtained from a dry eye subject (equivalent to Grade 3). 


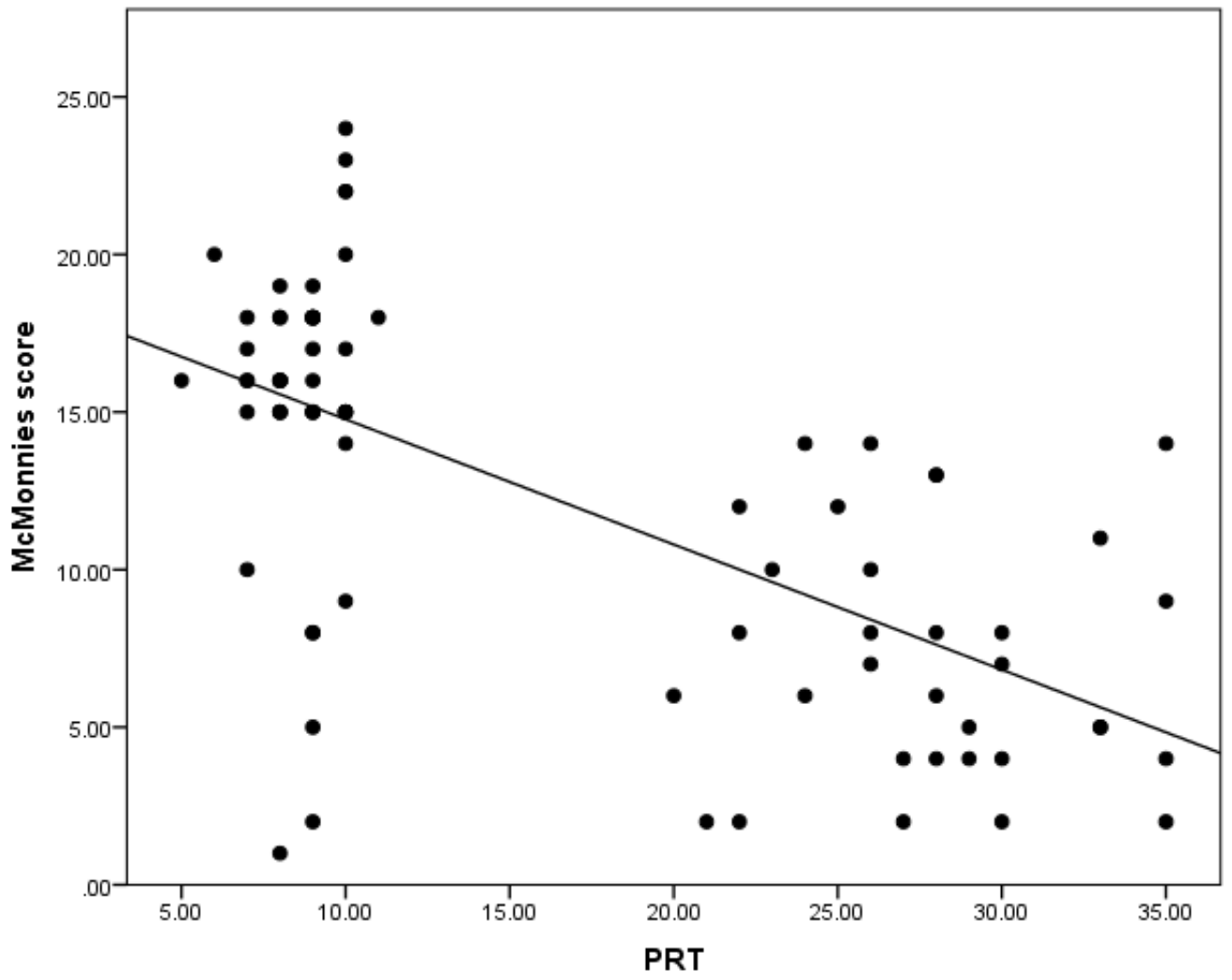

410 Figure 3. Correlation between PRT test and McMonnies score for all subjects.

411

412

413

414

415

416

417

418 


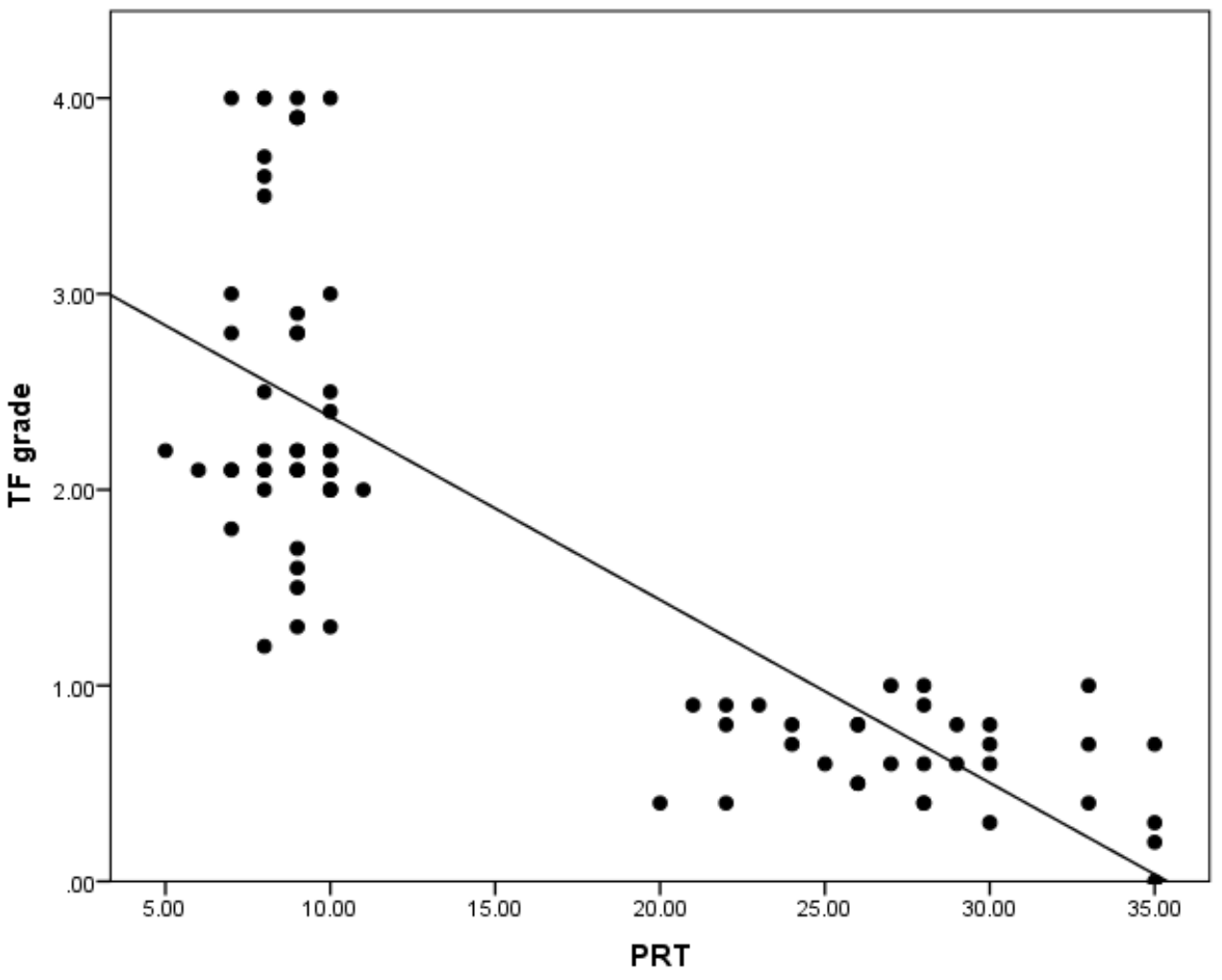

419

420

Figure 4. Correlation between tear ferning grade and PRT test for all subjects.

421

422

423

424

425

426

427

428 


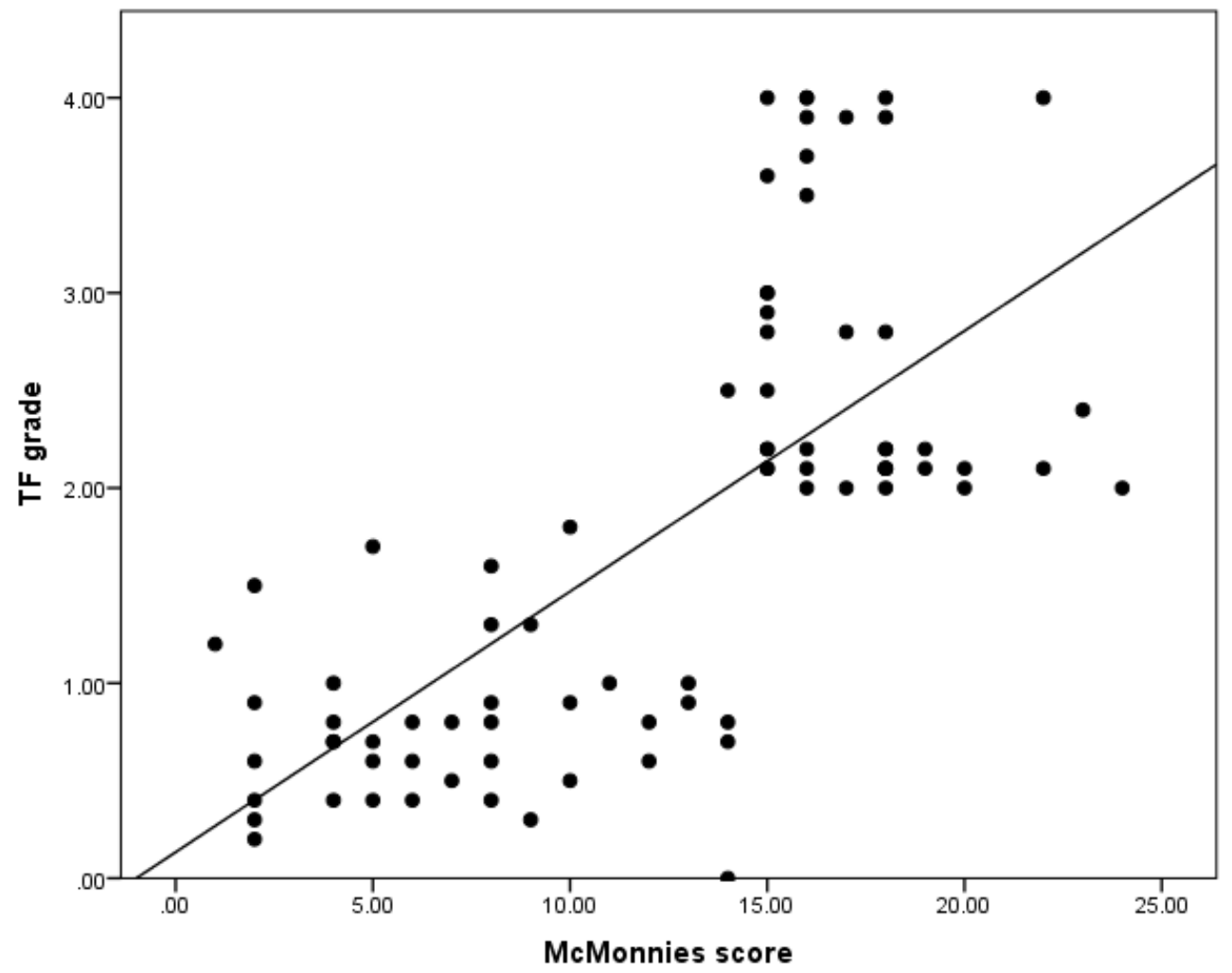

430 Figure 5. Correlation between tear ferning grade and McMonnies score for all subjects. 